\title{
INVERSIÓN EN EXPLORACIÓN Y EXPLOTACIÓN EN HIDROCARBUROS Y RECAUDACIÓN DEL IMPUESTO A LA RENTA EN PERÚ
}

\section{INVESTMENTS IN EXPLORATION AND EXPLOITATION IN HYDROCARBONS AND COLLECTION OF THE INCOME TAX IN PERU}

\author{
Bill Frank Oscanoa Ponce \\ Maestrista en Política y Gestión Tributaria con mención en Política y Sistema Tributario de la Universidad Nacional Mayor de San \\ Marcos - Senior de Impuestos en EY, experto especializado en el sector de Hidrocarburos y Energía, Lima, Perú. \\ Email: bill.oscanoa@gmail.com (Autor Corresponsal)
}

[Recibido: 20/06/2016 Aceptado: 16/08/2017]

\section{RESUMEN}

La investigación permitió establecer la relación entre las inversiones en exploración y explotación en hidrocarburos y la recaudación del Impuesto a la Renta en el Perú durante el periodo 2000-2016. A partir de dicho objetivo, se realizó un análisis descriptivo sobre la relación entre las variables y un análisis inferencial: el coeficiente de correlación de Pearson y la regresión lineal simple. Siendo así, se obtuvieron como principales resultados que existe una relación entre las inversiones en exploración y explotación en Hidrocarburos y la recaudación del Impuesto a la Renta, y que el grado de dicha relación es fuerte. Además, se constató que el sentido de la relación es positiva y estadísticamente significativa. De este modo, se concluyó que a mayores niveles de inversión en exploración y explotación en hidrocarburos mayores niveles de recaudación del Impuesto a la Renta. Es así que, se recomienda al Estado peruano en un contexto de crisis sectorial a establecer un Plan de Inversiones para que consecuentemente obtenga un nivel adecuado de recaudación del Impuesto a la Renta por el sector de hidrocarburos.

\section{PALABRAS CLAVE}

Inversión, impuesto a la renta, reglas de juego, crisis.

\begin{abstract}
The investigation allowed to establish the relation among the investments in exploration and exploitation in Hydrocarbons and the collection of the Income Tax in Peru during the period 2000-2016. Based on this objective, a descriptive analysis was performed on the relationship between the variables and an inferential analysis: the Pearson correlation coefficient and the simple linear regression. Thus, the main results obtained show that there is a relation among the investments in exploration and exploitation in Hydrocarbons and the collection of the Income Tax. The degree of this relation is strong, it has a positive sense and it is statistically significant. Thus, it was concluded that when higher levels of investment in exploration and exploitation in Hydrocarbons occur, it results in higher levels of income tax collection. Thus, it is recommended to the Peruvian State in a sectoral crisis context to establish an Investment Plan so that consequently obtain an adequate collection of Income Tax for the hydrocarbons sector.
\end{abstract}

\section{KEYWORDS}

Investment, income tax, rules of the game, crisis.

\section{INTRODUCCIÓN}

El presente estudio se enfoca en la relación entre las inversiones en exploración y explotación en hidrocarburos y la recaudación del Impuesto a la Renta en Perú. Para tal efecto, se formula la siguiente interrogante: ¿Existe relación entre las inversiones en exploración y explotación del sector Hidrocarburos y la recaudación del Impuesto a la Renta en el Perú durante el periodo 2000-2016?

Desde tal perspectiva, la hipótesis que responde a la pregunta es: "A mayores niveles de inversión en exploración y explotación del sector Hidrocarbu- ros mayores niveles de recaudación del Impuesto a la Renta en el periodo 2000-2016".

Para tal efecto, se exponen los fundamentos teóricos de la teoría del orden espontáneo y el orden construido sustentada por Hayek (2014), para explicar que las reglas de juego en una

Como Citar: Oscanoa, B. (2017). Inversiones en exploración y explotación en hidrocarburos y recaudación del impuesto a la renta en Perú. Quipukamayoc, 25(49), 81- 88. doi: http://dx.doi.org/10.15381/quipu.v25i49.14283 
sociedad se deben adecuar conforme evolucione el mercado; por otro lado, la teoría de las instituciones de North (1993), para fundamentar que las reglas de juegos impuestas en una sociedad deben promover la inversión y producción, para así como consecuencia obtener un buen desempeño económico como Estado.

Asimismo, se analizan los datos de la estadística petrolera del 2000-2016 publicado por Perupetro y así como de la información histórica del 20002016 de la recaudación del Impuesto a la renta por sectores publicado por la Superintendencia Nacional de Aduanas y Administración Tributaria (en adelante, "SUNAT").

Bajo tal premisa, el estudio recurre al enfoque cuantitativo, por ser probatorio a través de mediciones estadísticas. Por cierto, la principal limita-

Tabla 1.

Principales diferencias entre el orden espontáneo y orden construido Fuente: Rodríguez (2016)

\begin{tabular}{ll}
\multicolumn{1}{c}{ Orden Espontáneo } & \multicolumn{1}{c}{ Orden Construido } \\
Orden que se autogenera o endógeno & Orden que se construye o exógeno \\
\hline Formado por la evolución & $\begin{array}{l}\text { Creado artificialmente a través del diseño } \\
\text { humano }\end{array}$ \\
\hline $\begin{array}{l}\text { Proviene de acciones humanas no inten- } \\
\text { cionadas encaminadas a otros fines }\end{array}$ & $\begin{array}{l}\text { Proviene de planes intencionados (deli- } \\
\text { berados) }\end{array}$ \\
\hline $\begin{array}{l}\text { Carece de finalidad concreta (cuyo conte- } \\
\text { nido particular no es conocido o previsto } \\
\text { por nadie) }\end{array}$ & $\begin{array}{l}\text { Tiene fines concretos (mandados especí- } \\
\text { ficos) }\end{array}$ \\
\hline $\begin{array}{l}\text { Sustentado por el racionalismo evolu- } \\
\text { cionista (reconoce la abstracción como } \\
\text { único instrumento con el que nuestra } \\
\text { mente puede tratar una realidad cuya } \\
\text { plena comprensión se le escapa) }\end{array}$ & $\begin{array}{l}\text { Sustentado en el racionalismo cons- } \\
\text { tructivista (no reconoce los conceptos } \\
\text { abstractos son un medio para vencer la } \\
\text { complejidad de lo concreto) }\end{array}$ \\
\hline $\begin{array}{l}\text { Instituciones de mayor importancia: el } \\
\text { lenguaje, la sociedad, la costumbre, la } \\
\text { moral, la moneda, el trueque, el mercado, } \\
\text { la competencia, el liberalismo, el sistema } \\
\text { de precios y el Derecho. }\end{array}$ & $\begin{array}{l}\text { Concibe todas las instituciones como } \\
\text { resultados de planes deliberados: la fa- } \\
\text { empresa, el ejército y la legislación. }\end{array}$ \\
\hline $\begin{array}{l}\text { Griegos: utilizaron el término "cosmos" } \\
\text { para el orden formado por evolución. }\end{array}$ & $\begin{array}{l}\text { Griegos: utilizaron el término "taxis" para } \\
\text { el orden creado artificialmente. }\end{array}$ \\
\hline
\end{tabular}

ción de la investigación es el número de observaciones disponibles, el cual restringe el uso de análisis estadísticos más avanzados. Dado que se cuenta con una serie anual de corta duración no se utilizan otras variables que podrían enriquecer el modelo.

Para una mejor comprensión del tema propuesto, el contenido se ha estructurado en cuatro partes. La primera comprende la fundamentación. En la segunda se plantea la metodología. La tercera abarcan los resultados. En la cuarta se aborda la discusión. Luego, se presentan las conclusiones. Finalmente, se da a conocer las referencias bibliográficas.

\section{Teoría del Orden Espontáneo y Orden Construido}

Hayek (2014) aborda principalmente esta teoría haciendo la alusión que el orden que conocemos en la sociedad

ha sido fruto de un proceso histórico a lo largo de nuestra existencia como humanidad. El concibe dos conceptos fundamentales lo que denomina: el "Orden espontáneo" y el "Orden Construido", precisando que en toda sociedad existe al menos algún tipo de orden.

Por el cual, a lo largo del tiempo de la humanidad se han establecido ciertos ordenes espontáneos que surgieron fruto de la necesidades tales como el lenguaje, la moneda, el mercado, el derecho, entre otros que fueron evolucionando hasta convertirse en algunos casos de simple órdenes a hasta desarrollarse en un determinado orden construido.

En sentido, Hayek (2014) explica que un orden espontáneo es un sistema que se autogenera de manera evolutiva por las necesidades humanas mediantes sus acciones no intencionadas. Es un orden que no ha sido creado de forma deliberada a través del diseño humano; por lo que, no es susceptible de conocimiento completo por parte de los individuos, en donde se descubre o se desarrolla a través de un proceso constante de prueba y error, en el que la sociedad lo irán adaptando a sus necesidades sobre la base de los nuevos elementos que van adquiriendo, por ejemplo, conocimientos, valores, costumbres, normas, precios, etc. Por otro lado, Hayek (2014) nos argumenta que en cambio el orden construido es el resultado del diseño humano (racionalismo constructivista), en el que si existe una intención deliberada por parte del agente humano con el objetivo de obtener determinados fines específicos. Siendo así que, Hayek (2014) rechaza considerar al orden espontáneo como si fuera un orden construido a fin de no desnaturalizar su esencia como orden en la sociedad. 
En ese orden de ideas, podemos calificar al mercado como un orden espontáneo, ya que se autogenero como fruto de la necesidad humana de intercambiar y que se mantiene en constante evolución. Sin embargo, observamos con bastante frecuencia como los gobiernos intervienen (interfieren) en los mercados, en forma desmesurada, creando reglas de juego con ciertos privilegios que destruye la esencia del orden espontáneo. De ahí que, resulta relevante que los gobiernos al momento de legislar, no distorsionen el orden espontáneo del mercado, y que pretendan alcanzar sus fines concretos.

Por el contrario, las reglas de juego en vez de imponer al mercado, estos deben ir adaptándose en forma paulatina al mercado e ir evolucionado acorde a las necesidades del mercado, y no comportarse de forma estática, que entorpezcan las interacciones humanas.

\section{Teoría de las Instituciones}

North (1993) fundamenta la tesis de las Instituciones ("reglas de juegos") para explicar de qué manera las instituciones y los cambios institucionales afectan la economía, por lo que define a las instituciones como:

"Las reglas de juego de una sociedad o más formalmente, como las limitaciones ideadas por el hombre que dan forma a la interacción humana. Que en cierta forma estructuran incentivos en el intercambio humano, sea político, social o económico. Por lo que, el cambio institucional conforma el modo en que las sociedades evolucionan a lo largo del tiempo, siendo esto clave para entender el cambio histórico" (North, 1993, p.13).
Según North (1993), la función principal de las instituciones en la sociedad es:

“[...] es reducir la incertidumbre estableciendo una estructura estable (pero no necesariamente eficiente) de la Interacción humana. [...] Por lo que las instituciones definen y limitan el conjunto de elecciones de los individuos. [...] que pueden ser creadas como lo son las constituciones políticas de los Estados; o bien evolucionar simplemente a lo largo del tiempo, como evoluciona el derecho consuetudinario". (North, 1993, p.1416)

En sentido, todas las limitaciones que la sociedad pueda crear para dinamizar la interacción humana son denominadas instituciones los cuales se encuentran comprendidas por i) reglas formales (limitaciones o restricciones formales), ii) reglas informales (limitaciones restricciones informales) y iii) Características para garantizar su cumplimiento (ejecución obligatoria).

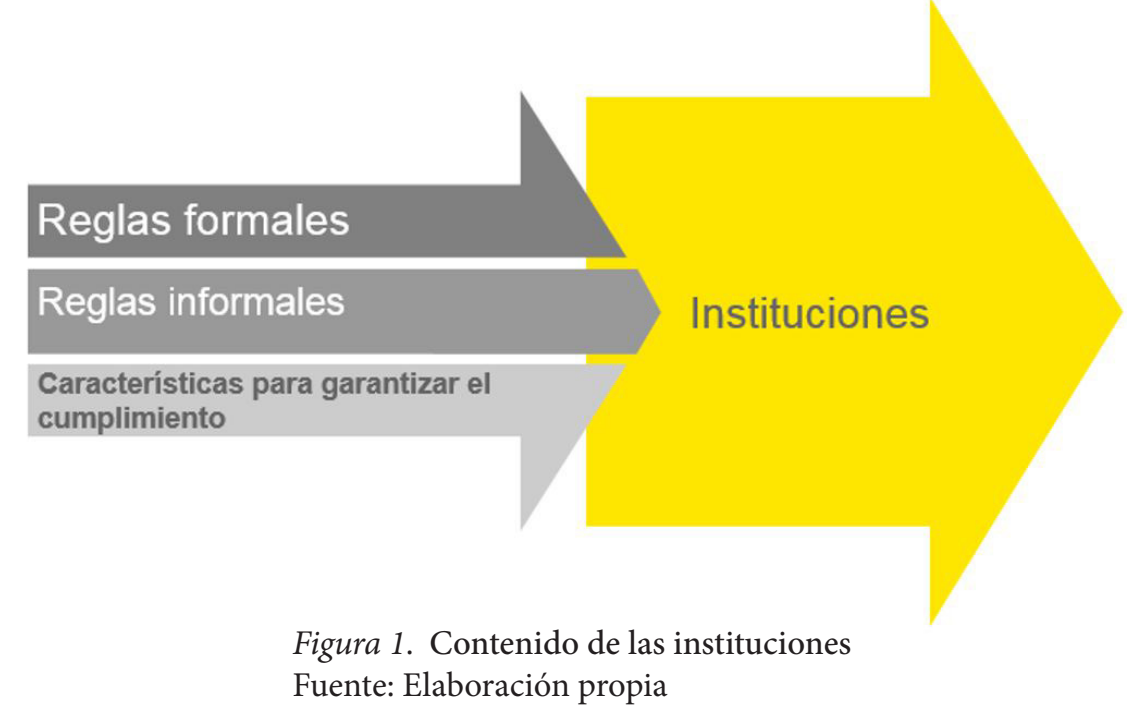

Siendo así, debemos conceptualizar a las limitaciones informales como aquellas reglas que no son escritas, que se van acumulando con el transcurso del tiempo y quedan registradas en los usos y costumbres (Rodríguez, 2016, p.203). En donde dicha información, es trasmitida socialmente $y$ son parte de la herencia que llamamos cultura - podemos definir la cultura como: "trasmisión de una generación a la siguiente por la enseñanza y la imitación de conocimientos, valores y otros factores - que influyen en la conducta" (North, 1993, p.55), proporcionándonos así un marco conceptual fruto de la evolución histórica de la interacción humana.
Consecuentemente, las reglas formales son el resultado del conjunto de limitaciones informales no escritas que han evolucionado en el contexto de interacción repetida (intercambio) entre los jugadores. Estos evolucionan para enfrentar problemas específicos de intercambio (North, 1993, p.59). Dicho de otro modo, según Rodríguez (2016):

"Las reglas formales son reglas escritas en normas legales y constituyen restricciones creadas por el hombre para resolver problemas específicos. En donde el cumplimiento de estas reglas es obligatorio y se requiere de un poder 
coercitivo para hacerlas cumplir -entiéndase que este tipo de instituciones son de dominio público" (p. 203)

En ese orden ideas, North (1993) concluye que la institución tiene efectos en el desempeño económico de un determinado Estado, el autor menciona:

[..] "Partiendo de una teoría de la conducta humana combinada con una teoría de los costos de negociación. Cuando las combinamos podemos entender por qué existen las instituciones y que papel desempeñan en el funcionamiento de las sociedades, y si agregamos una teoría de la producción podremos analizar las funciones de las instituciones en el desempeño de las economías". (North, 1993, p.43)

En otras palabras, las instituciones tienen efectos sobre los costos de producción, en donde su correcta implementación traerá consigo una disminución de los costos de transacción y transformación. Proporcionando una estructura de intercambio que promueva a los jugadores a realizar mayores actividades productivas que conlleva así un mejor desempeño económico como Estado.
Por lo que, podemos concluir que un país con un buen marco institucional diseñado con reglas de juegos que dinamice y desarrolle el sistema económico a un bajo costo obtendrá un crecimiento económico sostenido, basado en reglas de juegos que incentiven la inversión y producción de los agentes económicos en una determinada sociedad.

\section{Situación actual del sector de Hidrocar-} buros en el Perú: Crisis sectorial

En los años noventa, el sector de Hidrocarburos sufrió grandes cambios estructurales derivadas de modificaciones relevantes en el marco legal, específicamente en el año 1993, con la promulgación de la Nueva Ley Orgánica de Hidrocarburos (en adelante, "LOH). Posteriormente, en el transcurso de los años del 2000 a 2011, el Perú fue creciendo en los niveles de inversión, en exploración y explotación impulsados por las reformas legales - reglas de juegos - realizadas en décadas anteriores que dinamizaron el sector, principalmente impulsados por los inversionistas privados, que desde 1993 se vieron respaldadas principalmente por 1) la nueva Constitución Política del Perú del 1993 y 2) la promulgación de la LOH.

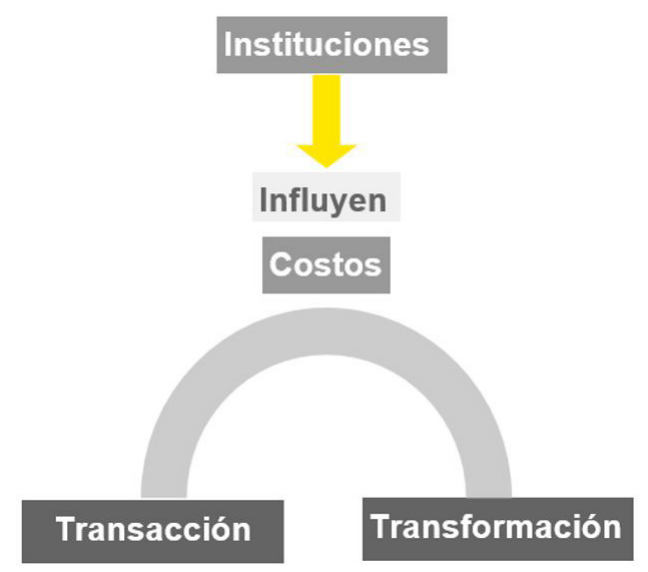

Figura 2. Efectos de las instituciones / Fuente: Instituciones, Cambio institucional y Desempeño económico. Elaboración propia

Por lo que, los niveles de inversión durante una primera etapa comprendida desde el 2000 al 2011 de acuerdo a datos oficiales de Perupetro fueron ascendentes. Evidenciando así, una tendencia de crecimiento sostenido en un marco legal de reglas de juegos que se promovió la inversión privada, precisando que se encontraba en un contexto del mercado del petróleo en auge. Como consecuencia de ello, el nivel de la recaudación del Impuesto a la Renta por el sector de Hidrocarburos durante dicho periodo se incrementó significativamente

No obstante, en una segunda etapa comprendida en los años 2012-2016, se evidencio una reducción del nivel de inversiones en los proyectos de exploración y explotación por parte de las empresas privadas en nuestro país. Trae como efecto menores niveles de recaudación del IR comprendidos entre el 2012 al 2016, enmarcado en un mismo marco legal - reglas de juegos estáticos - de años anteriores, pero en un contexto del mercado distinto a décadas anteriores.

En virtud a lo señalado anteriormente, se verifica que las reglas de juego fueron estáticas y no adecuadas al nuevo escenario del mercado, ya que según lo fundamentado teóricamente las Instituciones - reglas de juego deben ir evolucionado de forma paulatina al mercado; así como su implementación deben orientar a promover una mayor inversión y producción en un contexto coyuntural del mercado (orden espontáneo).

Más en dicha situación es necesario revisar las reglas de juegos y reformularlas para que se adecuen al nuevo contexto - crisis sectorial - en el mercado; recurriendo en ciertas circunstancias a recurrir a la experiencia internacional para poder afrontar el nuevo escenario. 
Teniendo en cuenta este contexto, es importante empezar con la reforma institucional para promover e incentivar las inversiones del sector hidrocarburos, introduciendo reglas de juegos beneficiosos que promuevan la productividad y generen en consecuencia mayores niveles de recaudación del Impuesto a la Renta de forma sostenida.

\section{MATERIAL Y MÉTODOS}

El estudio correspondió a un diseño no experimental, pues no se manipula la variable independiente.

La investigación fue correlacional porque tuvo como propósito evaluar la relación que existe entre las inversiones en exploración y explotación del sector Hidrocarburos y la recaudación del Impuesto a la Renta en el Perú durante el periodo 2000-2016.

El enfoque fue cuantitativo por ser probatorio a través de mediciones estadísticas.

\section{Unidad de análisis}

Datos de la estadística petrolera del 2000-2016 publicado por Perupetro, y así como de la información histórica del 2000-2016 de la recaudación del Impuesto a la renta por sectores publicado por la SUNAT.

Tratamiento de los datos y análisis de la información

A partir de los datos obtenidos de la data estadística petrolera del 20002016 e información histórica del 2000-2016 de la recaudación del Impuesto a la renta por sectores publicado por la SUNAT, se aplicaron las siguientes técnicas estadísticas y métodos de estimación:
- Análisis descriptivo para determinar la relación entre las inversiones en exploración y explotación del sector Hidrocarburos, y la recaudación del Impuesto a la Renta en el Perú durante el periodo 20002016.

- Análisis inferencial a través de pruebas estadísticas para verificar la existencia y sentido de relación entre las dos variables de interés.

Dentro del análisis inferencial, cabe precisar que la principal limitante del estudio fue la disponibilidad de datos, lo cual restringió el uso de análisis estadísticos más avanzados. Dado que se contó con una serie anual de corta duración, tampoco se utilizaron variables que podrían haber enriquecido el modelo. Al mismo tiempo, si bien los datos corresponden a series de tiempo, dado que conciernen al seguimiento de las variables a lo largo del tiempo, se optó por tratarlos como un corte transversal, el cual opera sobre la base que las observaciones son independientes del tiempo debido a que las estimaciones como una serie de tiempo de corta duración llevaría a inferencias que afectan la interpretación de los resultados. Por tal motivo, a efectos de comprobar la hipótesis se recurrieron a dos aproximaciones: el coeficiente de correlación de Pearson y la regresión lineal simple.

Coeficiente de correlación lineal de Pearson

Se verifico la existencia y sentido de relación entre las dos variables de interés mediante el coeficiente de correlación lineal de Pearson que se calculó de la siguiente manera:

En donde:

$$
\begin{gathered}
\rho_{X, Y}=\frac{\sigma_{X Y}}{\sigma_{X} \sigma_{Y}}=\frac{E\left[\left(X-\mu_{X}\right)\left(Y-\mu_{Y}\right)\right]}{\sigma_{X} \sigma_{Y}}, \\
\rho_{X, Y}=\frac{\sigma_{X Y}}{\sigma_{X} \sigma_{Y}}=\frac{E\left[\left(X-\mu_{X}\right)\left(Y-\mu_{Y}\right)\right]}{\sigma_{X} \sigma_{Y}},
\end{gathered}
$$

De manera que si el valor " $r$ " del índice de correlación varía en el intervalo $[-1,1]$, indicando el signo el sentido de la relación se interpreta lo siguiente:

- Si $r=1$, existe una correlación positiva perfecta. El índice indica una dependencia total entre las dos variables denominada relación directa, ya que cuando una de ellas aumenta, la otra también lo hace en proporción constante.

- Si $0<\mathrm{r}<1$, existe una correlación positiva.

- Si $r=0$, no existe relación lineal. Pero esto no necesariamente implica que las variables son independientes, debido a que pueden existir todavía relaciones no lineales entre las dos variables.

- Si $-1<\mathrm{r}<0$, existe una correlación negativa.

- Si $r=-1$, existe una correlación negativa perfecta. El índice indica una dependencia total entre las dos variables llamada relación inversa, ya que cuando una de ellas aumenta, la otra disminuye en proporción constante.

\section{RESULTADOS}

En la Tabla 2, se observa la evolución de las variables endógena (inversión en exploración y explotación del sector Hidrocarburos) y exógena (recaudación del Impuesto a la Renta) que se utilizaron para el análisis de las pruebas estadísticas. 
Tabla 2.

Inversión en exploración - explotación ejecuta del Sector Hidrocarburos y recaudación del Impuesto a la Renta 2000-2016 (MM PEN)

Fuente: Perupetro y SUNAT (2017). Elaboración propia

\begin{tabular}{cccc} 
Ejercicio & Inversión en Exploración & Inversión en Explotación & Recaudación del Impuesto a la Renta \\
\hline 2000 & 43 & 832 & 117 \\
\hline 2001 & 105 & 564 & 29 \\
\hline 2002 & 110 & 1237 & 158 \\
\hline 2003 & 42 & 1204 & 273 \\
\hline 2004 & 144 & 764 & 366 \\
\hline 2005 & 331 & 875 & 666 \\
\hline 2006 & 436 & 1764 & 792 \\
\hline 2007 & 751 & 2560 & 921 \\
\hline 2008 & 1120 & 3542 & 464 \\
\hline 2009 & 1559 & 1766 & 864 \\
\hline 2010 & 2098 & 1619 & 1474 \\
\hline 2011 & 1286 & 2384 & 1779 \\
\hline 2012 & 2415 & 2380 & 1908 \\
\hline 2013 & 1375 & 2663 & 1903 \\
\hline 2014 & 1500 & 2057 & 913 \\
\hline 2015 & 1080 & 1494 & 394 \\
\hline 2016 & 158 & 967 & \\
\hline
\end{tabular}

Análisis descriptivo de la relación entre las inversiones en exploración y explotación del sector Hidrocarburos y la recaudación del Impuesto a la Renta en el Perú.

\section{Inversión ejecutada del Sector Hidrocaburos 2000-2016 (MM PEN)}

$$
\begin{aligned}
& 6000 \\
& 5000 \\
& 4000 \\
& 3000 \\
& 2000 \\
& 1000
\end{aligned}
$$

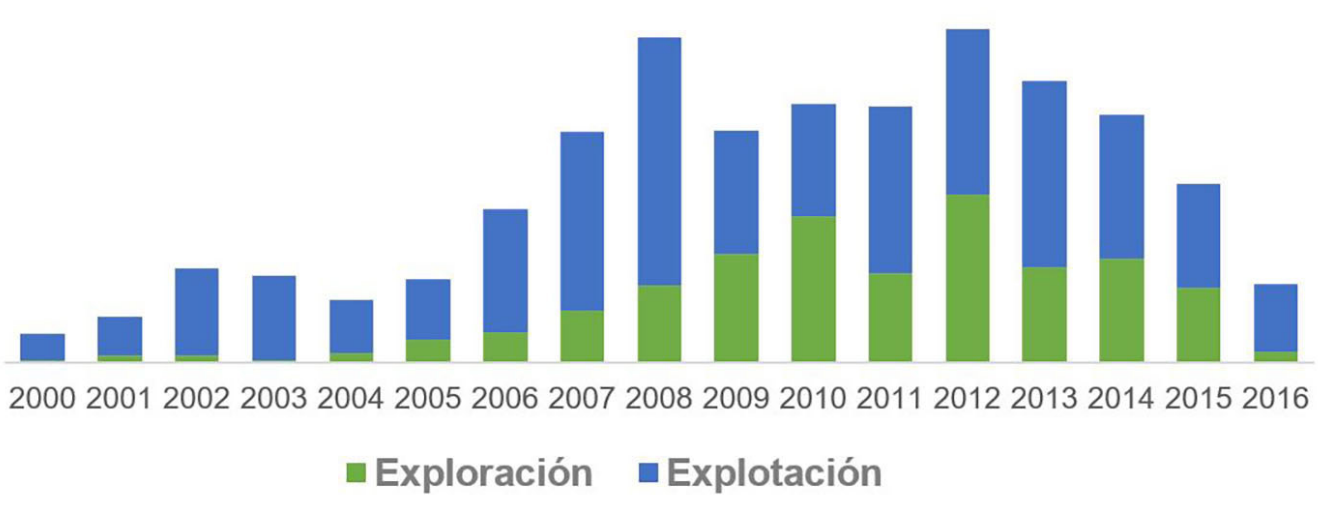

Figura 3. Inversión ejecutada del Sector Hidrocarburos, 2000-2016 Fuente: Perupetro. Elaboración propia 
Como se observa en la Figura 3, la tendencia en una primera etapa comprendida en los años 2000-2011 fue ascendente, respecto a la inversión en exploración y explotación, alcanzando en el 2000 un nivel de inversión de 425 MM PEN hasta llegar a unos
3,670 MM PEN en el 2011.

Como consecuencia, tal como se muestra en el Figura 4 el nivel de la recaudación del Impuesto a la Renta por el sector de hidrocarburos, du- rante dicho periodo, se comportó con una trayectoria ascendente similar al nivel de inversión en exploración y explotación, cuantificando en el 2000 unos 78,60 MM PEN de recaudación a unos 1,474MM PEN en el 2011.

\section{Recaudación del Impuesto a la Renta del Sector Hidrocarburos, 2000-2016 (MM PEN)}

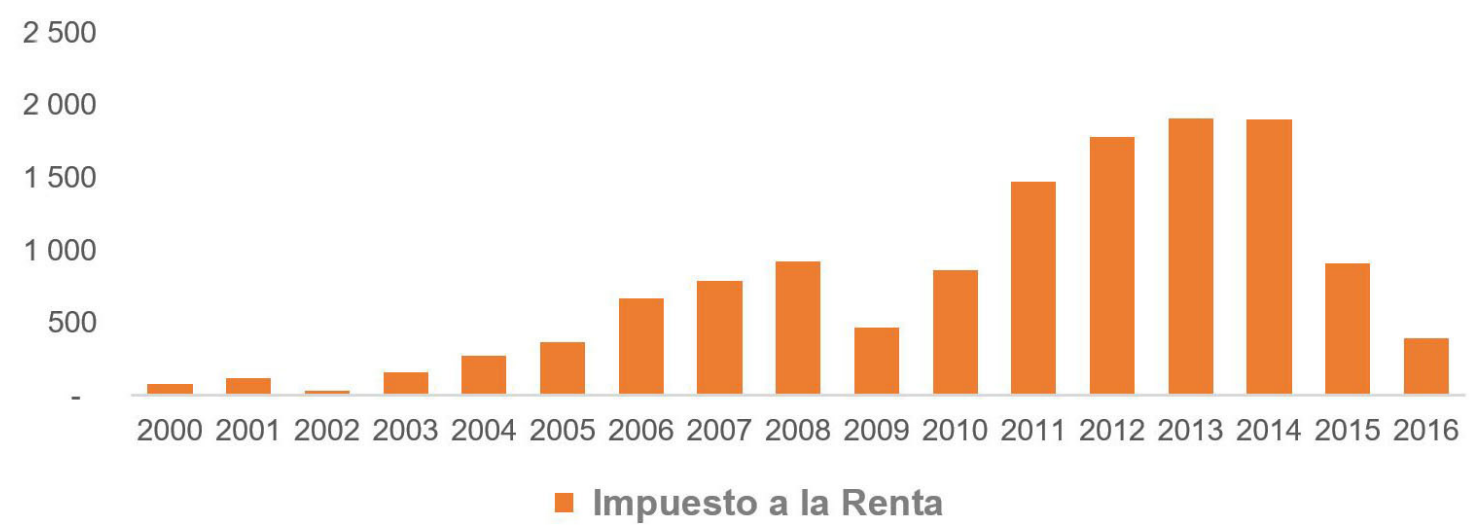

Figura 4. Recaudación del Impuesto a la Renta del Sector Hidrocarburos, 2000-2016

Fuente: SUNAT (2017). Elaboración propia

Asimismo, en la Figura 3 se verifica una segunda etapa comprendida en los años 2012-2016, donde se observa una tendencia negativa de la inversión, generando solo en el 2012 un nivel de inversión por 4, 796 MM PEN a ir cayendo en el 2014 en unos 3,357 MM USD hasta llegar a un 1,124 MM PEN en el 2016.

Por lo que, se contrasta una tenden-

cia negativa en la recaudación del Impuesto a la Renta, obteniendo en el 2012 una recaudación de unos S/.1779,38 MM, a pasar en el 2015 a unos S/.913,26 MM y llegar en el periodo 2016 aún S/.393,93 MM.

Análisis inferencial a través de pruebas estadísticas para verificar la existencia y sentido de relación entre las dos variables de interés.
Según los resultados obtenidos de la correlación de Pearson, se observa en la Tabla 3 un coeficiente de 0,823 , que representa una correlación positiva muy alta entre las dos variables de inversión en exploración y explotación del sector Hidrocarburos y la recaudación del Impuesto a la Renta en el Perú durante el periodo 2000-2016.

Tabla 3 .

Resultados de la Coeficiente Pearson

Fuente: Elaboración propia. Procesada en el programa estadístico SPSS

\begin{tabular}{|c|c|c|c|c|c|}
\hline $\mathbf{R}$ & & R cuadrado & R cuadrado ajustado & \multicolumn{2}{|c|}{ Error estándar de la estimación } \\
\hline ,823 & & ,677 &, 656 & \multicolumn{2}{|c|}{377,856} \\
\hline \multicolumn{3}{|c|}{ Coeficientes no estandarizados } & Coeficientes estandarizados & $\mathbf{t}$ & Sig. \\
\hline & $\mathrm{B}$ & Error estándar & Beta & & \\
\hline INVERSIÓN & 361 &, 064 & ,823 & 5,608 &, 000 \\
\hline (Constante) & $-138,827$ & 186,279 & &,- 745 & ,468 \\
\hline
\end{tabular}


Por lo que, de los resultados obtenidos se obtiene la siguiente fórmula para realizar las estimaciones del va-

\begin{tabular}{|l|}
\hline $\mathrm{Y}=-138827+0,361 \mathrm{X}$ \\
\hline $\mathrm{Y}=$ Constante \\
\hline $\mathrm{X}=$ Nivel inversión \\
\hline $\mathrm{Y}=$ Nivel de recaudación del Impuesto a la Renta \\
\hline
\end{tabular}

Asimismo, en la Figura 5 referido al diagrama de dispersión e Indicador de tendencia, se observa de la información procesada una tendencia po-

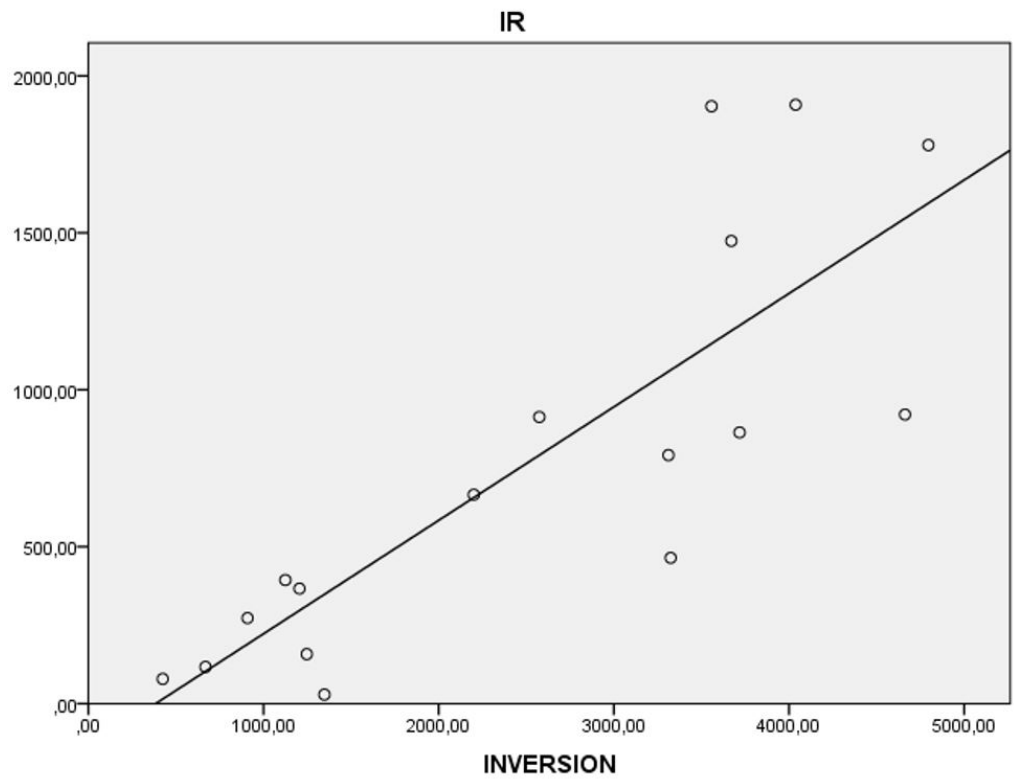

Figura 5. Diagrama de dispersión e indicador de tendencia

Fuente: Elaboración propia. Procesada en el programa estadístico SPSS

Por lo tanto, bajo el modelo estadístico de Pearson contrastamos que existe correlación positiva muy alta entre las inversiones en exploración y explotación del sector Hidrocarburos, y la recaudación del Impuesto a la Renta en el Perú durante el periodo 2000-2016.

\section{DISCUSIÓN}

Del análisis realizado, de acuerdo a lo datos estadístico de Perupetro y SUNAT, existe una coeficiente de Pearson por 0,823 que representa una

sitiva, ya que los datos analizados no se encuentran muy dispersos entre el conjunto de datos. de la otra variable (inversión). lor de una de ellas conociendo el valor

IR 